\title{
Relación Dinámica entre la Glucosa Intersticial y la Glucosa Plasmática Usando Modelos Matemáticos del tipo Entrada - Salida
}

\author{
Dynamic Relationship between Interstitial Glucose and Plasma Glucose Using \\ Input-Output Mathematical Models
}

\author{
Ángela María Lema-Pérez ${ }^{1}$, Laura Lema-Pérez², José García-Tirado ${ }^{3}$ \\ ${ }^{1,3}$ ITM Institución Universitaria, Medellín, Colombia \\ ${ }^{1}$ Facultad de Ciencias Exactas y Aplicadas, ${ }^{2}$ Universidad Nacional de Colombia, Medellín, Colombia \\ Facultad de Minas, Escuela de Procesos y Energía, \\ ${ }^{3}$ Facultad de Ciencias Económicas y Administrativas \\ angelalema99650@correo.itm.edu.co, llemap@unal.edu.co, josegarcia@itm.edu.co
}

\begin{abstract}
Resumen- La Diabetes Mellitus tipo 1 (T1DM por sus siglas en inglés) es una enfermedad autoinmune que afecta a gran cantidad de la población mundial. Esta enfermedad se caracteriza porque el páncreas ya no es capaz de producir la hormona insulina la cual es necesaria para la captación de energía de la mayoría de las células del cuerpo humano. Uno de los avances que se plantean actualmente para el tratamiento de esta enfermedad es por medio de un sistema de control automático de glucosa en sangre denominado páncreas artificial. Este sistema usa un monitor continuo de glucosa (CGM, por sus siglas en inglés) y una bomba de insulina que actúa por órdenes de un sistema de control. El CGM toma mediciones en el tejido intersticial abdominal cada 5 min lo que plantea un retardo con respecto a la glucosa plasmática que es la variable controlada. Este retardo es el causante de varios de los problemas de los sistemas de control que actualmente se plantean. En este estudio se pretende relacionar ambas mediciones a través de un modelo matemático de primer orden, en el cual la glucosa plasmática es la entrada y la glucosa intersticial es la salida. El modelo fue identificado, a partir datos reales de un paciente, mediante la curva de reacción. Los parámetros $k, \tau$ y $d$ del modelo, fueron ajustados comparando los parámetros calculados en seis modelos identificados, logrando llegar a un único modelo que relaciona matemáticamente ambas mediciones de glucosa.
\end{abstract}

Palabras clave- diabetes mellitus, glucosa intersticial, glucosa plasmática, insulina, modelo de primer orden, monitoreo de glucosa.

Abstract- Type 1 Diabetes Mellitus (T1DM) is an autoimmune disease affecting most of the world population. This disease appears when the pancreas is no longer able to produce the insulin hormone which is needed to the energy intake by most of the body cells in the human being. One of the most promising treatments to this disease is by means of an automatic control system of the glucose concentration known as artificial pancreas. This artificial system uses a continuous glucose monitor (CGM) and an insulin pump which acts based on the control system. The CGM takes measurements each $5 \mathrm{~min}$ from the abdominal interstitium which in fact is a delayed measurement of the blood glucose. This delay causes many issues in the design of control systems. In this study both measurements are related through a first order mathematical model, in which the blood glucose is considered the input and the interstitial glucose is considered the output. A model was identified from real patient data and by using the reaction curve methodology. The model parameters $k, \tau$ y $d$ were fitted by comparing the parameters of six identified mathematical models, and a unique mathematical model which relates both blood glucose measurements and interstitial glucose measurements was found.

Key Words - blood glucose, diabetes mellitus, first order model, glucose monitoring, interstitial glucose, insulin.

\section{INTRODUCCIÓN}

Actualmente, la Diabetes Mellitus es considerada una epidemia mundial según datos de la Organización Mundial de la Salud [1]. Esta misma organización señala la evolución de esta enfermedad en las últimas dos décadas reflejándose en altos costos humanos, sociales y económicos. Según el Atlas de la Diabetes presentado por la OMS, en el 2013 había 382 millones de personas con esta condición, cifras que tienden a 
incrementar de manera exponencial en los próximos años [1]. Con el ánimo de proveer un tratamiento más eficiente en comparación al manejo actual de la enfermedad [1], existe una iniciativa de algunas universidades y centros de investigación del mundo hacia la creación de un páncreas artificial que permita restablecer el mecanismo homeostático de la glucosa que fue dañado por la enfermedad. Este páncreas artificial es, a grandes rasgos, un dispositivo electrónico de infusión inteligente de insulina (y en algunos casos glucagón) que basa su inteligencia artificial en un sistema avanzado de control automático de glucosa.

La variable de interés (variable a controlarse) es la concentración de glucosa en plasma. Sin embargo no existen dispositivos comerciales de medición de ésta variable en línea. La única manera no clínica de medir esta variable mediante glucómetros, los cuales son dispositivos de uso ambulatorio que usan sangre capilar de los dedos de la mano para efectuar la medición de glucosa en sangre por medio de una tirilla reactiva. La otra forma de medir la glucosa es clínica, la cual se lleva a cabo haciendo pasar la sangre del paciente por medio de un catéter y luego a un medidor en línea, pero al utilizar esta forma, el paciente requiere ser hospitalizado. Una de las razones para la no existencia de dispositivos de medición de glucosa en plasma comerciales es que deben ser invasivos y por ende se aumentan las posibilidades de infección.

Por otro lado, se disponen de CGM's, los cuales son dispositivos que miden glucosa en el intersticio del abdomen. La concentración de glucosa que se mide en dicho intersticio es a partir de la glucosa que ha logrado difundirse desde el torrente sanguíneo a través de pequeños capilares. Por lo tanto, debido al tiempo de difusión de glucosa, la medida que se hace en el intersticio no es la misma que se hace en plasma. Debido a esto, hay una necesidad de relacionar la glucosa intersticial con la glucosa plasmática, ya que utilizando el CGM se tienen mediciones en línea y sin necesidad de hospitalización.

La T1DM requiere de una terapia intensiva de insulina y un estricto control de la glucosa en sangre. El tratamiento convencional de este tipo de diabetes implica inyecciones regulares de diferentes clases de insulina [2]. Los pacientes miden su concentración de glucosa en sangre usando un medidor externo, y según el valor obtenido, calculan la dosis de insulina que se deben inyectar. Un tratamiento alternativo es el uso de sistemas automatizados de administración de insulina en conjunto con un monitor continuo de glucosa (CGM, por sus siglas en inglés).

En la Fig. 1 se observa las diferentes capas que debe atravesar el CGM para sensar los datos de glucosa intersticial. El retardo se ve evidenciado, ya que debe atravesar el tejido subcutáneo y el músculo.

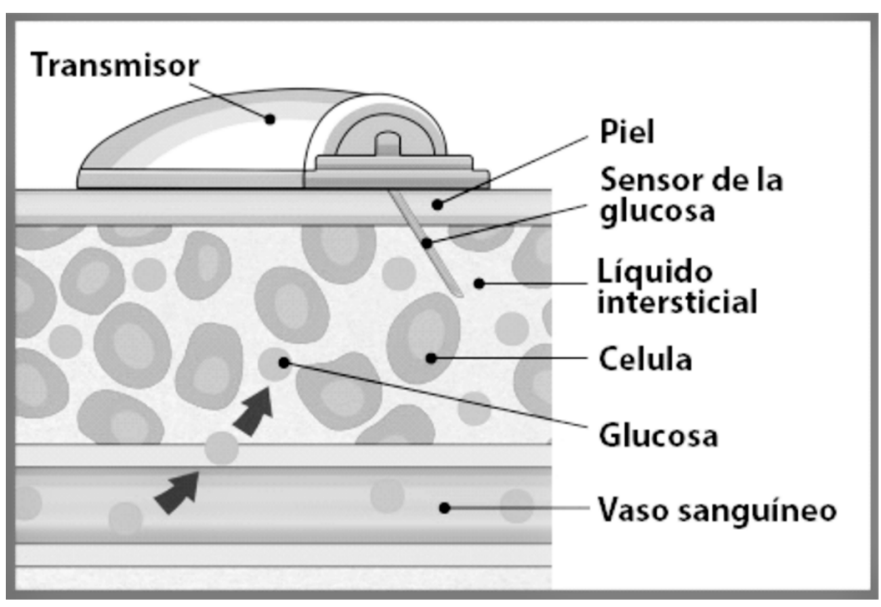

Fig. 1. Tiempo de difusión CGM [16]

A mediados del siglo XX se comenzó a estudiar exhaustivamente la T1DM desde la Ingeniería de Control [3]. Especialmente se ha venido trabajando en la construcción de un páncreas artificial externo que mejore la calidad de vida del paciente, permitiendo un control más estricto de los niveles de glucosa en sangre. Es este sentido, han habido avances pertinentes en el campo del monitoreo continuo de la glucosa en seres humanos, los cuales han dejado un desarrollo significativo desde la creación de dispositivos externos que miden glucosa en sangre, conocidos como glucómetros, hasta la creación de monitores mínimamente invasivos que miden la glucosa en el tejido subcutáneo, conocidos como CGM [4]. Este último es un elemento clave para tener un registro de la dinámica de la glucosa de cada paciente, según las situaciones en las que se encuentra a diario. El CGM realiza mediciones de glucosa cada 5 minutos en el tejido subcutáneo, con un retardo de aproximadamente 15 minutos [5], con respecto a las mediciones obtenidas con un medidor externo de glucosa (glucosa en plasma). Lo anterior hace que se tengan series de tiempo de la glucosa intersticial, lo que a su vez se convierte en un indicador de la evolución dinámica de la glucosa en plasma, pero con una incertidumbre que debe tenerse en cuenta a la hora de tomar decisiones de control [6]. En este sentido el trabajo acoplado y sincronizado entre la bomba de insulina y el CGM dan como resultado un páncreas artificial externo [7], siempre y cuando se busque la regulación autónoma de la concentración de glucosa.

El monitoreo de la glucosa es una parte integral del control de la diabetes, y mantener la concentración de glucosa en sangre dentro del rango establecido, es la única manera de prevenir complicaciones de la diabetes que amenacen la vida de quien 
padece esta enfermedad. En consecuencia, los retos que involucra el CGM desde el campo tecnológico y de caracterización dinámica son todavía materia de investigación por científicos alrededor del mundo [8]. Por lo tanto, es importante comparar la respuesta dinámica, por lo menos desde modelos matemáticos del tipo entrada-salida, de la relación de la glucosa plasmática y la glucosa intersticial.

Los CGM surgieron hace aproximadamente 30 años [9] , siendo en su mayoría mínimamente invasivos. Estos dispositivos proporcionan una serie de tiempo detallada de las fluctuaciones de glucosa en el tejido intersticial. La realimentación de dicha información en pacientes con T1DM [10], ha sido utilizada para mostrar un aspecto positivo en el control de la glucemia, incluyendo la reducción de la variabilidad de la glucosa, hipoglucemias nocturnas e hiperglucemias. Sin embargo, varios estudios realizados han concluido que, a pesar de varios años de desarrollo, la tecnología de los monitores continuos de glucosa sigue teniendo retos en términos de sensibilidad, estabilidad, calibración y en la identificación de la relación dinámica existente entre las concentraciones de glucosa plasmática y glucosa intersticial [11]. Cabe resaltar que la variable de interés es la concentración de glucosa medida en plasma, y sensarla continuamente implica un tratamiento más invasivo y riesgoso para el paciente, que medirla en el espacio intersticial mediante un CGM. Éste dispositivo, presenta un retardo en la medición con respecto a los valores tomados de un glucómetro. Adicionalmente según la literatura revisada, hay poco estudio acerca de la relación dinámica entre la glucosa intersticial y la glucosa plasmática [12]. Asimismo, se ha evidenciado que no se ha propuesto relaciones del tipo entradasalida, por medio de funciones de transferencia para describir la relación entre estas dos dinámicas, lo cual es materia de estudio en el presente artículo. Debido a esto, se pretende mostrar una relación dinámica existente entre la glucosa intersticial y la glucosa plasmática, dada la dificultad de ejecutar acciones de control directamente desde la información entregada por el CGM. Se identificaron seis modelos del tipo entrada-salida entre la glucosa intersticial y la glucosa plasmática usando datos de monitoreo (CGM) actualmente disponibles.

El resto del artículo es como sigue. En la Sección II se explican los materiales y métodos llevados a cabo para la identificación del modelo matemático. Los resultados obtenidos y su respectiva discusión se presentan en la Sección III. Finalmente, en la Sección IV se presentan las conclusiones y el trabajo futuro a partir del desarrollo de este trabajo.

\section{MATERIALES Y MÉTODOS}

El método de la curva de reacción es un método experimental propuesto por Ziegler, Nichols y Rochester en 1942 para la identificación de modelos de funciones de transferencia de primer orden con tiempo muerto de sistemas dinámicos [13], [14]. Este método se hizo muy popular en el siglo pasado pues permitió la creación de varios métodos de sintonía de controladores PID basados en el modelo identificado siempre y cuando la planta no tenga integradores y sea estable en lazo abierto [15].

El método consiste en la excitación de la planta real por medio de una entrada tipo escalón para generar una respuesta en la salida o variable que desea controlarse, como se muestra en la Figura 2. Del análisis de estas señales de entrada y salida se identifican la ganancia de lazo abierto, la constante de tiempo y el tiempo muerto, denominados en adelante como $k, \tau$ y $d$, respectivamente, las cuales se relacionan por medio de (1) donde, además de las variables ya definidas, $y(t)$ y $u(t)$ son la la salida y la entrada, respectivamente, y $s$ es la variable compleja de Laplace.

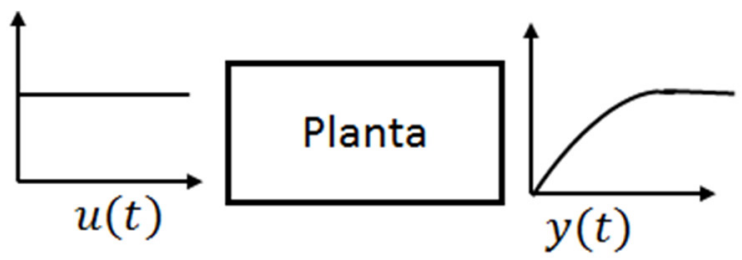

Fig. 2. Respuesta de la planta al aplicar un escalón unitario

$$
\frac{y(t)}{u(t)}=\frac{k}{\tau s+1} e^{-d s}
$$

La identificación de los tres parámetros del modelo (1) se hace como sigue. Primero se traza una línea tangente a la curva de respuesta en el punto de máxima pendiente. Esta línea se intersecta con el eje del tiempo y con el estado estacionario alcanzado, tal y como se ve en la parte inferior de la Figura 3. El tiempo muerto $d$ es el tiempo transcurrido desde que se aplicó la excitación hasta la intersección de la recta tangente con la línea de tiempo. La constante tiempo $\tau$ resulta restar el tiempo donde se intersectan la recta tangente con el nuevo estado estacionario con el tiempo muerto. La ganancia de lazo abierto $k$ se encuentra dividiendo el cambio en la salida $\Delta y$ con el cambio en la entrada $\Delta u$.

Con el objetivo de identificar un modelo matemático de primer orden que relacione las mediciones de glucosa intersticial con las mediciones de glucosa plasmática, se tomaron mediciones de estas dos variables de un paciente con T1DM usando como sensores un monitor continuo de glucosa CGM y un glucómetro, respectivamente. Las curvas de los datos fueron generadas en Matlab® y analizadas visualmente para 
seleccionar uno o varios tramos de curva que tenían un comportamiento similar al de un sistema dinámico lineal de primer orden con retardo. Mediante este método se identificaron los parámetros de seis modelos matemáticos tipo entrada-salida descritos por medio de (1). Una vez obtenidos los modelos matemáticos, se simularon en Simulink y cada respuesta obtenida se comparó con el respectivo tramo de curva seleccionada.

En la Figura 4 se muestra la serie de tiempo de aproximadamente $24 \mathrm{~h}$ de monitoreo mediante CGM para el paciente con T1DM. Nótese que se muestra con líneas punteadas la región en donde idealmente debería permanecer la concentración de glucosa en un paciente con T1DM. En este sentido, debajo de la línea inferior se dice que el paciente se encuentra en estado de hipoglucemia y por encima de la línea superior en estado de hiperglucemia. Ambos estados son indeseables en un paciente. En esta misma Figura, los óvalos punteados corresponden a dos de los tramos seleccionados para aplicar el método de identificación antes descrito.

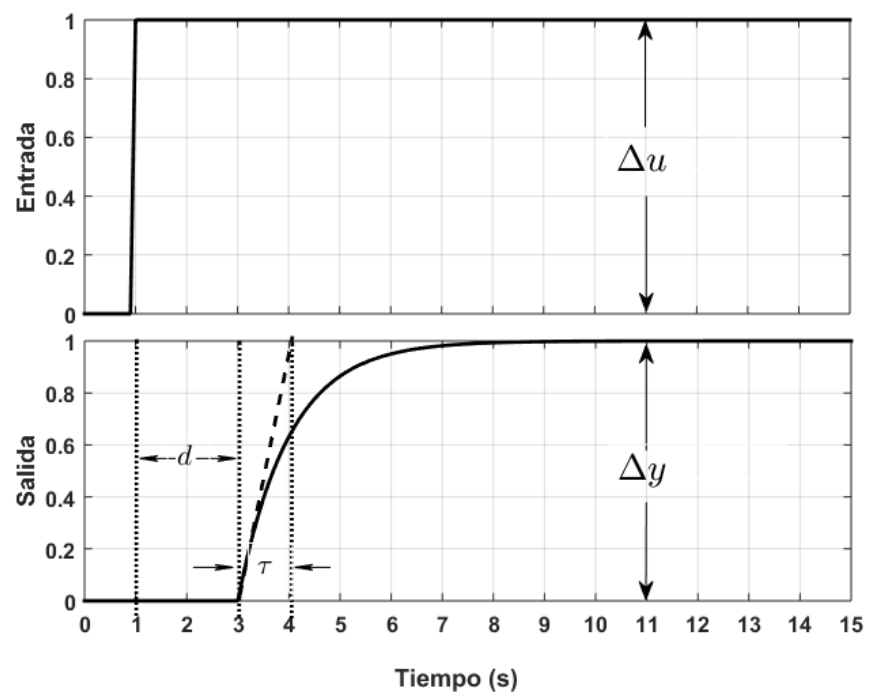

Fig. 3. Parámetros de la curva de reacción

El procedimiento se aplica teniendo en cuenta las mediciones de glucosa plasmática en los horarios del tramo de curva seleccionada. Se supone una entrada tipo escalón porque es una variable que reacciona mucho más rápido y de la cual se tienen sólo valores puntuales por las glucometrías. La ganancia $k$ es un parámetro de sensibilidad del sistema dinámico, representada por el cambio en la glucosa intersticial IG, dividida por el cambio en la entrada, representada por la glucosa plasmática BG, como se muestra en (2).

$$
k=\frac{\Delta y}{\Delta u}=\frac{\Delta I G}{\Delta B G}
$$

donde $\Delta I G$ es el cambio en la glucosa intersticial y $\Delta B G$ es el cambio den la glucosa plasmática. La ecuación de la línea recta también se usa para hallar la constante de tiempo:

$$
y-y_{1}=m\left(x-x_{1}\right)
$$

donde $m$ es la pendiente de la recta, $\left(x_{1}, y_{1}\right)$ son las coordenadas de un punto conocido adyacente al punto de interés y $(x, y)$ son las coordenadas del punto de interés, siendo $y=\Delta_{y} * 0.632$. En este sentido, se halla primero la pendiente de la recta con dos puntos conocidos, luego, con la pendiente, la ordenada y uno de los puntos conocidos se halla la abscisa, es decir, el tiempo de respuesta cuando el sistema alcanza el 63\% de la respuesta total. En la parte inferior de la Figura 3 se aprecia que tal resultado indica que la línea de razón máxima de cambio interseca la línea de valor inicial en $t=d$, y a la línea de valor final en $t=d+\tau$.

\section{RESULTADOS Y DISCUSIÓN}

Las gráficas reportadas en esta Sección muestran el comportamiento obtenido de los seis modelos identificados. En la Figura 5 se observan las seis gráficas diferentes, cada una incluye un tramo de curva de glucosa intersticial tomada de un monitoreo continuo de glucosa en un paciente real, la respuesta del modelo identificado a partir de ese mismo tramo y la entrada tipo escalón que fue ingresada y que representa la glucosa plasmática. En dichas curvas se puede notar que las pendientes del modelo identificado son muy similares a las pendientes de los tramos originales.

En la Tabla 1 se reportan los parámetros calculados para cada modelo identificado, con el fin de detectar el rango en el que se mueve cada uno y ajustar así los valores a un único modelo que relacione las dos mediciones de glucosa para un paciente determinado. Se observa que la respuesta del sistema alcanza un $63 \%$ de la respuesta total aproximadamente entre 0.26 y 0.7 $h$. Los valores de tiempo muerto o retardo son muy aproximados entre sí, obteniéndose casi en todos los tramos analizados un $d$ igual a $0.1611 \mathrm{~h}$. Mientras tanto, la ganancia o el nivel de sensibilidad del sistema, $k$, estuvo alrededor de 1 . 

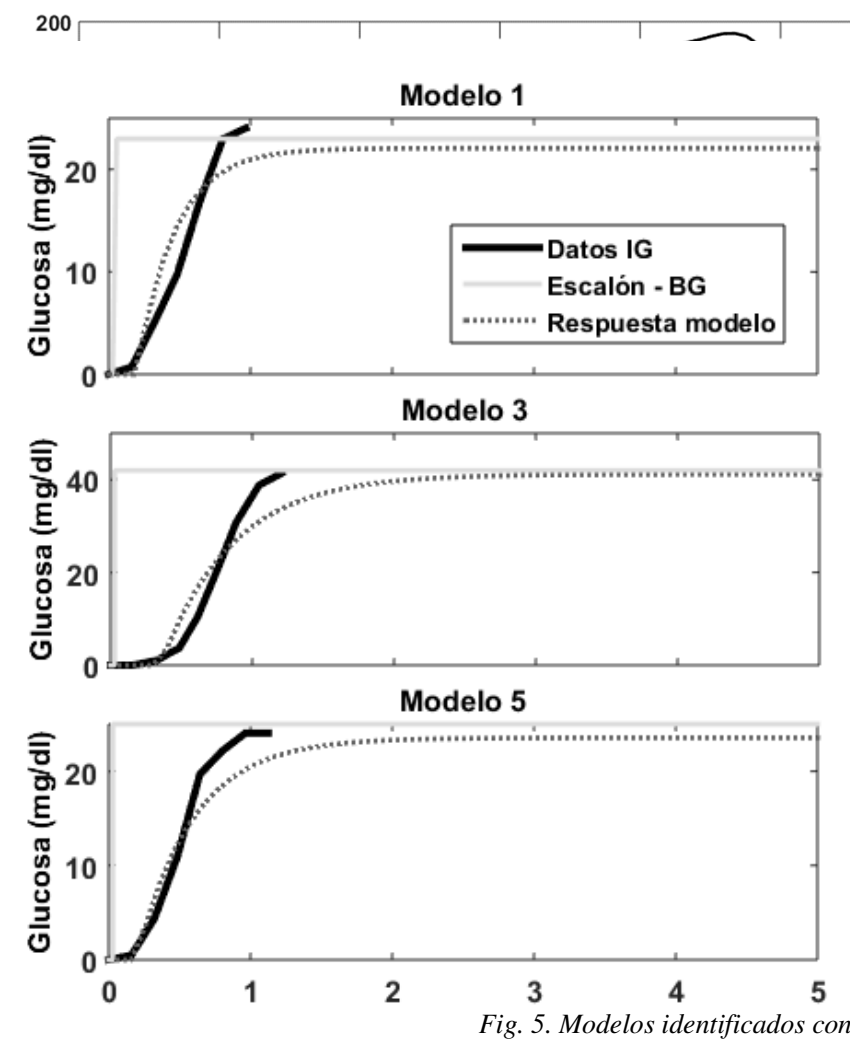

Tabla 1. Parámetros de los modelos identificados.

Promediando los valores de $\tau, d$ y $k$ se obtienen los parámetros de un único modelo que relaciona las mediciones de glucosa intersticial con las mediciones de glucosa plasmática. Dicho modelo se muestra en (3).

$$
\frac{I G}{B G}=\frac{K}{\tau s+1} e^{-d s}=\frac{0.964}{0.5 s+1} e^{-0.19 s}
$$

\begin{tabular}{|c|c|c|c|}
\hline Modelo & $\boldsymbol{\tau}(\boldsymbol{h})$ & $\boldsymbol{d}(\boldsymbol{h})$ & $\boldsymbol{k}$ \\
\hline 1 & 0.2689 & 0.1611 & 0.96 \\
\hline 2 & 0.539 & 0.161 & 0.93 \\
\hline 3 & 0.498 & 0.322 & 0.98 \\
\hline 4 & 0.589 & 0.171 & 0.99325 \\
\hline 5 & 0.395 & 0.161 & 0.942 \\
\hline 6 & 0.7006 & 0.161 & 0.983 \\
\hline
\end{tabular}

El modelo obtenido en (3) es de mucha utilidad dado que puede usarse para predecir la glucosa en plasma a partir de los datos entregados por un CGM. En este sentido, mediante (3) pueden completarse modelos matemáticos más complejos del ciclo homeostático de la glucosa para una posible implementación clínica de un control automático de glucosa o páncreas artificial.
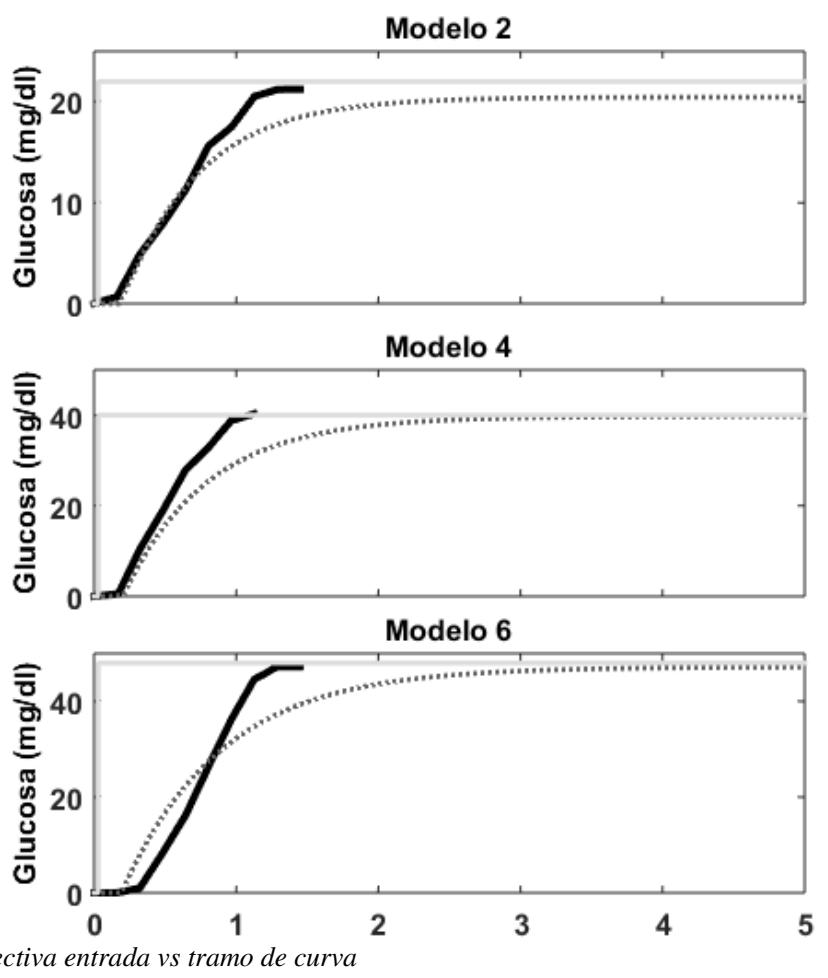

III. CONCLUSIONES Y TRABAJO FUTURO

Este estudio permite concluir que es posible hallar una relación matemática entre la medición de glucosa en plasma y la medición de la glucosa intersticial. Dicha relación matemática es simple, pues está representada por medio de una función de transferencia de primer orden que consta de tres parámetros $\tau, t_{0}$ y $\mathrm{K}$. Los parámetros del modelo fueron ajustados dentro de rangos estrechos a partir de la curva de reacción aplicada a diferentes tramos de curva que contenía los datos reales tomados de un paciente con T1DM. Esto indica que el método de la curva de reacción provee resultados prometedores para analizar la relación entre las dos variables estudiadas.

La entrada tipo escalón en la mayoría de los modelos fue ajustada a partir de datos de auto monitoreo del paciente y las gráficas de glucosa intersticial. Dado que los auto monitoreos no se realizan con la misma frecuencia que se hacen las mediciones de glucosa en el intersticio, obtener valores exactos en las glucometrías que coincidan con los datos reportados en las gráficas de glucosa intersticial es complejo, por lo tanto se ve afectada la ganancia del sistema.

La relación matemática encontrada servirá principalmente para estudiar el retardo en las mediciones de glucosa intersticial con respecto a las mediciones de glucosa plasmática y poder estimar la concentración de glucosa en plasma a partir de las mediciones de glucosa en el intersticio. 
Como trabajo futuro se va a repetir el estudio usando varios sujetos incluyendo sujetos sanos y pacientes con T1DM y T2DM (Diabetes Mellitus tipo 2 por sus siglas en inglés). Se obtendrán mediciones de CGM y mediciones de glucosa en plasma cada 5 minutos usando métodos clínicos. Con esta información se espera concluir de forma más contundente con el hallazgo reportado en esta publicación.

\section{REFERENCIAS}

[1] M. George Bakris, F. Lawrence Blonde, MD, M. Andrew J.M. Boulton, P. Mary de Groot, M. Eddie L. Greene, M. Robert Henry, F. Sherita Hill Golden, MD, MHS, P. Frank Hu, MD, MPH, P. Derek LeRoith, MD, M. Robert G. Moses, P. Stephen Rich, M. Matthew C. Riddle, M. Julio Rosenstock, M. William V. Tamborlane, R. Katie Weinger, EdD, R. Judith WylieRosett, EdD, E. Board, M. Nicola Abate, M. Silva Arslanian, P. Angelo Avogaro, MD, F. Ananda Basu, MD, P. John B. Buse, MD, M. Sonia Caprio, D. Robert Chilton, F. Kenneth Cusi, MD, FACP, P. Paresh Dandona, MD, M. Stefano Del Prato, P. Dariush Elahi, P. Franco Folli, MD, M. Robert G. Frykberg, DPM, M. W. Timothy Garvey, M. Ronald B. Goldberg, F. Margaret Grey, DrPH, RN, M. Richard Hellman, F. Rita Rastogi Kalyani, MD, MHS, F. Rory J. McCrimmon, MBChB, MD, F. Harold David McIntyre, MD, M. Sunder Mudaliar, M. Gianluca Perseghin, M. Anne L. Peters, M. Jonathan Q. Purnell, M. Peter Reaven, M. Helena Wachslicht Rodbard, P. Pedro Romero-Aroca, M. David J. Schneider, M. Elizabeth R. Seaquist, M. Norbert Stefan, M. Jeff Unger, P. Ram Weiss, MD, Ms. Deborah J. Wexler, $\mathrm{MD}, \mathrm{Bc}$. Joseph Wolfsdorf, MD, and P. Tien Yin Wong, MBBS, FRCSE, FRANZCO MPH, "January Supplement Combined_Final Diabeets Care," J. Clin. Appl. Res. Educ., vol. 38, no. January, p. 99, 2015.

[2] D. Owens, A. H. Barnett, J. Pickup, D. Kerr, P. Bushby, and D. Hicks, "Blood glucose self-monitoring in type 1 and type 2 diabetes: reaching a multidisciplinary consensus," Diabetes Prim Care, vol. 6, no. 1, pp. 816, 2004.

[3] C. Cobelli, C. D. Man, G. Sparacino, L. Magni, G. De Nicolao, and B. P. Kovatchev, "Diabetes: Models , Signals , and Control," vol. 2, pp. 54-96, 2009.

[4] S. K. Vashist, "Continuous Glucose Monitoring Systems: A Review," Diagnostics, vol. 3, no. 4, pp. 385-412, 2013.

[5] B. S. M. E. Philip J. Stout, M.S., Nina Peled, Ph,D. Brian J. Erickson, B.S.M.E., Michael e. Hilgers, B.S. Joel R. Racchini, M.S. and Thomas B. Hoegh, "Comparison of Glucose Levels in Dermal Interstitial Fluid and Finger Capillary Blood," DIABETES Technol. Ther., vol. 3, no. 1, pp. 81-90, 2001.

[6] C. Wei, D. J. Lunn, C. L. Acerini, J. M. Allen, A. M. Larsen, M. E. Wilinska, D. B. Dunger, and R. Hovorka,
"Measurement delay associated with the Guardian® RT continuous glucose monitoring system," Diabet. Med., vol. 27, no. 1, pp. 117-122, 2010.

[7] A. Manuscript, "New-generation diabetes management:glucose sensor-augmented insulin pump therapy," vol. 8, no. 4, pp. 449-458, 2012.

[8] N. Poolsup, N. Suksomboon, and A. M. Kyaw, "Systematic review and meta-analysis of the effectiveness of continuous glucose monitoring ( CGM ) on glucose control in diabetes," Diabetol. Metab. Syndr., vol. 5, no. 1, p. 1, 2013.

[9] P. Rossetti, J. Bondia, J. Vehí, and C. G. Fanelli, "Estimating plasma glucose from interstitial glucose: The issue of calibration algorithms in commercial continuous glucose monitoring devices," Sensors, vol. 10, no. 12, pp. 10936-10952, 2010.

[10] T. Koschinsky, K. Jungheim, and L. Heinemann, "Glucose Sensors and the Alternate Site Testing-Like Phenomenon: Relationship Between Rapid Blood Glucose Changes and Glucose Sensor Signals," Diabetes Technol. Ther., vol. 5, no. 5, pp. 829-843, 2003.

[11] M. Breton and B. Kovatchev, "Analysis, modeling, and simulation of the accuracy of continuous glucose sensors," J. Diabetes Sci. Technol., vol. 2, no. 5, pp. 853-862, 2008.

[12] E. R. a Y. K. Kulcu and J. A. a T. Amada, "Physiological Differences Between Interstitial Glucose and Blood Glucose Measured in Human Subjects," Diabetes Care, vol. 26, no. 8, pp. 24052409, 2003.

[13] Ziegler J.G., N. B. Nichols, and N. Y. Rochester, "Optimum Settings for Automatic Controllers," Trans. ASME, vol. 64, pp. 759-768, 1942.

[14] M. King, Process Control: A Practical Approach, 2nd ed. 2016.

[15] C. A. Smith and A. B. Corripio, Control Automático de Procesos. Teoría y Práctica, Primera ed. México D.F.: EDITORIAL LIMUSA, S.A. de C.V., 1991.

[16] Medtronic, "Cómo calibrar su sensor," Medtronic, 2014. . 